\title{
LOS REFORMULADORES DE RECAPITULACIÓN EN EL CORPUS PRESEEA DE GRANADA*
}

\section{REFORMULATORS OF RECAPITULATION IN THE PRESEEA CORPUS OF GRENADE}

\author{
Natalia Ruiz GonZÁlez \\ Universidad de Granada \\ nataliaruiz@ugr.es \\ https://orcid.org/0000-0002-3496-6933
}

Recibido: $21 / 10 / 2019$

Aceptado: 30/01/2020

\begin{abstract}
Resumen
La reformulación recapitulativa presenta en un nuevo miembro discursivo una síntesis o resumen de un elemento expuesto previamente o una serie de ellos. Lo más común es que se lleve a cabo mediante la marcación discursiva y esta tiende a variar según el lugar y las características sociales de los hablantes que la emplean. Para comprobar cómo es el funcionamiento de estas unidades y su distribución social en el español de Granada, hemos llevado a cabo un estudio sociolingüístico basado en el corpus Preseea de la ciudad andaluza. Los resultados arrojan que la edad y el nivel educativo se revelan como factores clave en el uso de los recapitulativos, siendo superior cuanto más años y mayor nivel de estudios posee el hablante. Además, en fin es el reformulador preferido para cumplir esta función, abarcando casi un
\end{abstract}

\begin{abstract}
Recapitulative reformulation presents a new discursive member as a synthesis or summary of a previous element or a series of them. Commonly, this is done through discursive markers and, this varies depending on the place and social characteristics of the speakers who employ it. To check how these units are operating and their social distribution in Granada, we have carried out a sociolinguistic study on the PRESEEA corpus of this Andalusian city. The results show that age and educational level are key factors in the use of recapitulatives, higher in older speakers and with a higher level of studies. In addition, en fin is main marker to complete this function, covering almost $40 \%$ of the cases in which this group of discursive markers is used.
\end{abstract}

* Este trabajo se enmarca dentro del proyecto de investigación competitivo ECOPASOS-Granada financiado por MINECO/FEDER (Ref. FFI2015-68171-C5-2-P).

** Natalia Ruiz-González es beneficiaria de un contrato predoctoral de la Universidad de Granada a través de la ayuda de formación de profesorado universitario del Ministerio de Ciencia y Universidades (FPU15-05294).

Para citar este artículo / To cite this article: Ruiz González, Natalia (2020). Los reformuladores de recapitulación en el corpus Preseea de Granada. ELUA, 34: 193-212. https://doi.org/10.14198/ ELUA2020.34.9

Enlace / Link: https://doi.org/10.14198/ELUA2020.34.9 
$40 \%$ de los casos en que aparece este grupo de marcadores discursivos.

PALABRAS CLAVE: reformuladores, recapitulación, español de Granada, PreseEA, en fin.
KEY WORDS: reformulators, recapitulation, Spanish of Grenade, PrEsEeA, en fin.

\section{INTRODUCCIÓN}

El estudio de los marcadores discursivos en el discurso oral ha aumentado significativamente en los últimos años, pues su distribución es claramente distinta a la que se da en la escritura (Portolés Lázaro 2014: 126). Así, podemos encontrarnos con unidades más propias o frecuentes de la oralidad y otras características de los textos escritos. En el primer grupo encontramos elementos como bueno, en fin, o sea, vamos u hombre; y en el segundo, en síntesis, no obstante, a saber o por lo demás.

Esta distinción es especialmente visible en los reformuladores, a los que el hablante recurre para modificar parte de su discurso anterior porque considera que no se ha efectuado de la forma más eficaz y adecuada. Nuestra intención es estudiar el comportamiento pragmático y la distribución social de los reformuladores que se dedican a la recapitulación, es decir, aquellos que presentan en una nueva formulación, un resumen o conclusión de una serie de elementos precedentes. Todo ello se llevará a cabo a través del corpus Presees de la ciudad de Granada, partiendo de la base de que toda elección de un marcador, como la de cualquier otro fenómeno lingüístico, puede estar condicionada por la pertenencia del hablante a un grupo sociocultural determinado (Cortés Rodríguez 2008: 152).

Nuestro objetivo principal es estudiar cómo se distribuyen sociopragmáticamente los reformuladores recapitulativos en el español de la ciudad de Granada, donde creemos que se produce un uso preferencial por los hablantes de tercera generación y nivel de instrucción alto, ya que estos son más conscientes de la importancia de cerrar el discurso y dejar el contenido de la enunciación totalmente claro para el oyente. Además, creemos que, entre los marcadores discursivos que realizan esta función en el corpus de la ciudad de Granada, hallaremos unidades lingüísticas que las clasificaciones tradicionales no registran y que en la oralidad toman valores diferentes según el contexto en el que se integren. Por último, queremos averiguar si existe un marcador que presente mayor frecuencia de uso respecto al resto para cumplir esta función y qué hablantes lo emplean con mayor asiduidad.

Pese al notorio aumento de los estudios sobre marcadores del discurso en las últimas dos décadas ${ }^{1}$, el grueso del análisis que se ha realizado sobre este tipo de unidades ha girado en torno a su definición y clasificación, y no a su distribución espacial y social dentro de una comunidad de habla (Carbonero Cano y Santana Marrero 2010: 499). Contamos con trabajos de corte sociolingüístico sobre los marcadores discursivos en general en ciertos núcleos urbanos, como los de Martín Zorraquino (1991) sobre Zaragoza, Jorgensen y Martínez López (2007) sobre el habla juvenil de Madrid y Santana Marrero (2017) acerca del habla de Sevilla, dentro de España, y Said-Mohand (2014) sobre el español de Estados Unidos, Martín Butragueño (2003) en el español de México, González Mafud y Perdomo Carmona (2018) del habla de La Habana, Rodríguez Cadena (1999) y Vásquez Cantillo (2005, 2009) acerca del habla de Barranquilla, Rincón (2013) sobre Bucaramanga, Travis

1 Según datos de Vázquez Veiga, hasta un 3270,27\% (2011-2012: 5). 
(2005) sobre el español de Colombia, Cuartas López (2011) sobre Cartagena de Indias y Serna Pinto y Hernández Miranda (2016) respecto la ciudad de Pereira en Colombia, Domínguez y Álvarez (2005) y Martínez Matos y Domínguez (2006) acerca de Mérida (Venezuela), Taibo Cao (2016) sobre el español de Montevideo y Poblete Bennett y Cepeda (1997, 2006) acerca del habla de Valdivia, así como los trabajos sobre el habla culta que se incluyen en Valencia Espinoza (2014) y Valencia Espinoza y Vigueras Ávila (2015); y sobre reformuladores, como Rojas Inostroza et al. (2012), San Martín Núñez (2013, 2014, 2015, 2016a, 2016b, 2017) y San Martín Núñez y Guerrero González (2016) acerca del español de Santiago de Chile y Guirado Zapata (2019) sobre la ciudad de Caracas. Sin embargo, poco o nada se ha señalado de lo que sucede en el área oriental andaluza, a la que dedicamos este estudio.

\section{MARCO TEÓRICO}

Este trabajo se incluye dentro de los conceptos teóricos y metodológicos de la sociolingüística variacionista (Labov 1983), que concibe la variabilidad y heterogeneidad como un rasgo inherente de la lengua, aunque no de manera aleatoria, sino reglada (Silva-Corvalán y Enrique-Arias 2017: 268). Esta variación sociolingüística vendría condicionada por factores internos y por factores externos al lenguaje, como la edad, el sexo o el nivel sociocultural de los hablantes (Moreno Fernández 2015: 36). Además, a nuestro modo de ver, se trata de un tipo de variación que puede aplicarse a cualquier nivel de la lengua, incluido el plano discursivo, en el que se encuentran los marcadores del discurso. Asimismo, y siguiendo lo expuesto por Cortés Rodríguez (2008), estos elementos lingüísticos pueden analizarse cuantitativamente mientras consideremos que dos o más partículas tienen equivalencia semántico-pragmática en determinados contextos, con lo cual pueden interpretarse como dos formas de comunicar lo mismo.

Para nuestro trabajo tomamos en consideración la descripción de los marcadores aportada por Martín Zorraquino y Portolés Lázaro (1999) y Portolés Lázaro (2014), así como el rasgo de la polifuncionalidad que algunos autores atribuyen a estas unidades como Vásquez Cantillo (2005: 39), Garcés Gómez (2014: 17) o Fuentes Rodríguez (2015: 2). Dentro de este amplio y diverso grupo de unidades, los reformuladores constituyen el grupo de marcadores que permiten al hablante, y en algunos casos también a su interlocutor, volver sobre un fragmento del discurso para explicarlo, matizarlo, resumirlo o corregirlo (Garcés Gómez 2003: 112) si este así lo considera oportuno, siendo el nuevo miembro reformulado el que el oyente ha de considerar como legítimo (Martín Zorraquino y Portolés Lázaro 1999: 4121; Portolés Lázaro 2014: 109).

En su clasificación, Martín Zorraquino y Portolés Lázaro (1999) y Portolés Lázaro (2014) distinguen entre reformuladores explicativos, rectificativos, de distanciamiento y de recapitulación o recapitulativos. Estos últimos, en los que nos centraremos, presentan el miembro del discurso que introducen como una conclusión o recapitulación a partir de un elemento anterior o una serie de ellos, que pueden encontrarse expresos o implícitos (Garcés Gómez 2008: 115-116). Por tanto, "no se pone en cuestión la primera formulación en cuanto al contenido de los segmentos que la integran, sino que la segunda parte es el resultado sintético, objetivo o subjetivo, de una visión englobadora de lo considerado en los segmentos anteriores" (Garcés Gómez 2006a: 1317). 
Según Martín Zorraquino y Portolés Lázaro, este tipo de marcadores de reformulación puede conservar la misma orientación argumentativa de los miembros anteriores: en suma, en conclusión, en resumen y en síntesis; presentar miembros con la misma orientación o con una opuesta, pudiendo actuar en este caso como operadores, como en resumidas cuentas, en definitiva, a fin de cuentas, en fin y total; o mostrar una conclusión antiorientada, mucho más cercanos estos a los operadores, como ocurre con después de todo y al fin y al cabo (1999: 4133-34).

Además de estos marcadores, encontramos otros que, en determinados contextos, pueden actuar como reformuladores de recapitulación. Es el caso de los explicativos es decir y $o$ sea, que, combinados con la conjunción que, toman valores conclusivos cercanos a en fin que, presentando la consecuencia del enunciado anterior (Santos Río 2003 s.v. o sea; Briz Gómez et al. 2008 s.v. o sea que; Domínguez García 2016: 51; Murillo Ornat 2016: 250) y que ya demostramos en un trabajo anterior sobre el empleo de o sea en Granada (Ruiz-González 2018: 192-193). Y ocurre también con marcadores que Martín Zorraquino y Portolés Lázaro atribuían al grupo de enfocadores de alteridad, ya que, en su origen, apuntan directamente al oyente o a ambos interlocutores (1999: 4171). Hablamos, en este caso, de marcadores como bueno (Briz Gómez 2014: 33; Fuentes Rodríguez 1990: 140) y vamos (Figueras 2000: 304; Fuentes Rodríguez 2009 s.v. vamos; Portolés Lázaro 2014: 143), especialmente cuando este aparece acompañado de que (Briz Gómez 1997: 73; 2014: 224). También se incluyen otros marcadores menos frecuentes como lo cual o en efecto (Martí Sánchez 2013: 85-86), sin perder este último su principal función como mostrador de conformidad.

Pero, dentro de este grupo, en fin y total constituyen los recapitulativos propios del discurso oral o de textos escritos de carácter menos formal (Garcés Gómez 2017: 296), sobre todo el primero, que ha sido considerado la unidad más utilizada con el valor de reformulador de recapitulación en la lengua hablada (Martín Zorraquino y Portolés Lázaro 1999: 4137; Garcés Gómez 2006b: 343) y la que más funciones y valores ha desarrollado (Fuentes Rodríguez 1990: 151), actuando también como ordenador de cierre (Fuentes Rodríguez 2009 s.v. en fin; Domínguez García 2014), como continuativo y, derivado de este uso, como muletilla (Martí Sánchez 2013: 83). Es, por tanto, buen ejemplo de la polifuncionalidad que anunciábamos anteriormente para estas unidades (Adam y Revaz 1989: 97).

\section{CUESTIONES METODOLÓGICAS}

Esta investigación se enmarca dentro del Proyecto para el Estudio Sociolingüístico del Español de España y América (PRESEEA), un macroproyecto cuyo objetivo principal es construir un corpus sincrónico del español hablado en las principales ciudades de España y América, mediante una metodología común que permita el intercambio científico y la comparación de resultados (Moreno Fernández 1996: 258) sobre fenómenos lingüísticos que abarcan todos los niveles de la lengua.

Dentro del proyecto, se han manejado tres variables sociales como decisivas a la hora de elaborar el corpus. Estas son: la edad, en la que se distinguen tres generaciones, de 20 a 34 años, de 35 a 54, y de 55 en adelante; el sexo, aunque "en un buen número de análisis se ha revelado como una variable de escasa capacidad explicativa" (274); y el nivel de instrucción, dentro del cual se distinguen tres subvariables: grado de instrucción 
bajo (hablantes sin estudios o con estudios primarios), medio (personas con estudios secundarios) o alto (formación universitaria o superior).

Para aplicar el cuestionario se llevó a cabo un muestreo por cuotas de fijación uniforme, según el cual el universo de la población se divide en unas categorías determinadas y se asigna una cuota a cada uno de los distintos estratos (Moreno Fernández 2015: 312). En este caso, la cantidad asignada fue de tres informantes para cada una de las celdas, lo que hace un total de 54 informantes. Esto, en una ciudad con 237.929 habitantes en el momento de las entrevistas, supone un $0,0279 \%$, o, lo que es lo mismo, un informante por cada 4.406 habitantes (Moya Corral 2007: 43). Esta distribución puede observarse en el cuadro 1.

\begin{tabular}{|l|c|c|c|c|c|c|c|}
\cline { 2 - 7 } \multicolumn{1}{c|}{} & \multicolumn{2}{c|}{$\begin{array}{c}\text { Generación 1 } \\
\text { (19-34 años) }\end{array}$} & \multicolumn{2}{c|}{$\begin{array}{c}\text { Generación 2 } \\
\text { (35-54 años) }\end{array}$} & \multicolumn{2}{c|}{$\begin{array}{c}\text { Generación 3 } \\
\text { (más de 55 años) }\end{array}$} & \multirow{2}{*}{ Total } \\
\cline { 2 - 7 } \multicolumn{1}{c|}{} & Hombres & Mujeres & Hombres & Mujeres & Hombres & Mujeres & \\
\hline Nivel alto & 3 & 3 & 3 & 3 & 3 & 3 & 18 \\
\hline Nivel medio & 3 & 3 & 3 & 3 & 3 & 3 & 18 \\
\hline Nivel bajo & 3 & 3 & 3 & 3 & 3 & 3 & 18 \\
\hline Total & 9 & 9 & 9 & 9 & 9 & 9 & 54 \\
\hline
\end{tabular}

Cuadro 1. Distribución de los informantes en el muestreo por cuotas de fijación uniforme

Las grabaciones se realizaron entre 2005 y 2009 siguiendo los parámetros de PRESEEA, es decir, constituyen conversaciones semidirigidas o parcialmente dirigidas, ya que este tipo de entrevista "garantiza la tensión comunicativa y cierta homogeneidad temática" (Moreno Fernández 2015: 314). Fueron realizadas mediante el uso de un guion previo y a partir de unos módulos temáticos concretos que intentaban ofrecer la máxima confianza al interlocutor para que se expresara lo más espontáneamente posible (Moya Corral 2007: 44), buscando así reducir al mínimo la paradoja del observador (Labov 1972: 113).

Después de un rastreo detallado de cada grabación y su correspondiente transcripción, se encontraron 319 ocurrencias de marcadores con función de reformulación recapitulativa, en los que se centrará el posterior análisis de este trabajo. Su identificación no resultó fácil al tratarse de una variable de tipo pragmático-discursiva y por la polifuncionalidad que ya hemos apuntado como propia de los marcadores del discurso, que dificulta, a su vez, su reconocimiento y análisis.

De hecho, la concepción de una variable sociolingüística más allá del plano fonético ha sido duramente criticada por algunos estudiosos, sobre todo tras el trabajo de Lavandera (1978), que puso en duda este tipo de estudios amparándose en la imposible equivalencia semántica entre las variables. Sin embargo, según Moreno Fernández (2015: 21), la variación lingüística, entendida como dos formas distintas de decir lo mismo, se produce en todos los niveles de la lengua, desde el fonético hasta el discursivo.

Para detectar las unidades con el valor semántico de recapitulación, hemos aplicado la prueba de conmutación propuesta por Portolés Lázaro (2014: 79-84). Así, tomaremos en consideración todas aquellas unidades que presenten la información precedente concentrada 
en una nueva formulación y que sean fácilmente sustituibles por el marcador en fin y no por otro propio de la ordenación discursiva de cierre, como por último o finalmente.

Para su tratamiento estadístico hemos utilizado el programa SPSS, en la versión 20 para Windows 10. El grado de significación lo hemos definido en el 5\%, según lo cual, si p valor es menor de 0,05 será estadísticamente significativo. Hemos realizado para ello la prueba paramétrica Análisis de varianza (ANOVA) y la no paramétrica ANOVA de Kruskal Wallis, previendo que la distribución de los datos pudiera ser anormal. Para el análisis multivariable, sin embargo, usamos las tablas de contingencia y la prueba de Chi cuadrado de Pearson ${ }^{2}$.

Asimismo, teniendo en cuenta que la distribución de los tokens puede venir condicionada por características de los hablantes externas a la lengua o por la duración media de cada una de las entrevistas, hemos ponderado los datos para reajustar ese desequilibrio y que los resultados obtenidos fueran lo más fiables posibles ${ }^{3}$.

\section{RESULTADOS Y DISCUSIÓN}

\subsection{Resultados generales}

En nuestro corpus se ha identificado un total de 1186 tokens de marcadores reformuladores, que se distribuyen tal como puede observarse en la figura gráfica 1.

Como podemos percibir, el grupo de los reformuladores con función explicativa domina claramente en la discursividad de los granadinos de la muestra, lo cual revela la preferencia de los hablantes por realizar paráfrasis de sus discursos previos con vistas a la correcta interpretación del oyente, más que a presentar giros argumentativos o información nueva.

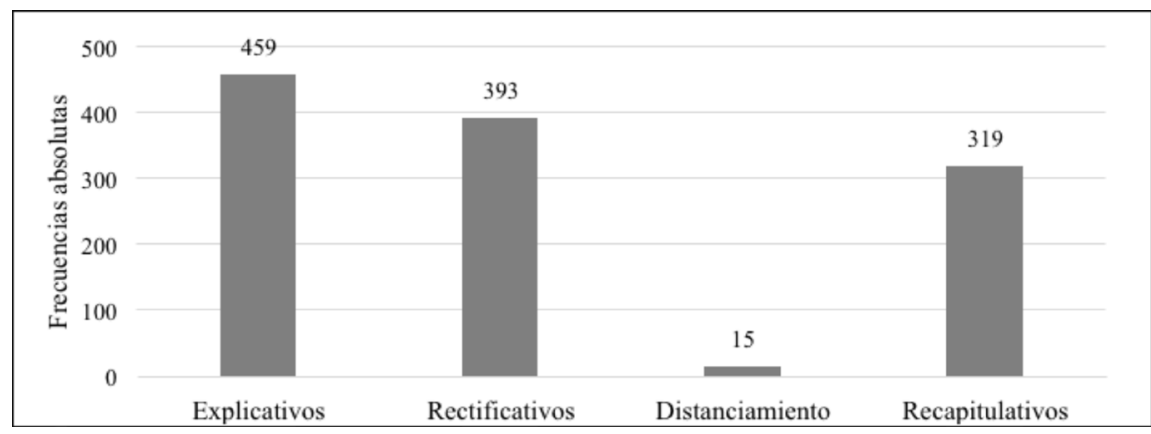

Fig. 1. Distribución porcentual de los marcadores de reformulación en la ciudad de Granada

Los reformuladores con función recapitulativa constituyen el tercer grupo de uso, después de los de función rectificativa, alcanzando 319 tokens o, lo que es lo mismo, un $26,90 \%$ de empleo en el total del corpus. Los reformuladores de distanciamiento, en cambio, carecen de total relevancia en el análisis de este tipo de unidades, contrariamente a lo

2 Esta es una prueba que mide la diferencia entre una distribución observada y la esperada y nos ayuda a probar la independencia de dos variables entre sí (Herrera Soler, Martínez Arias y Amengual 2011: 183).

3 Algunas grabaciones son ligeramente más largas que otras y hay hablantes que, personalmente, tienden más al uso de marcadores discursivos, con lo cual la ponderación nos ayuda a otorgar el mismo peso a todos los casos. 
que ocurre en otras ciudades como Santiago de Chile (San Martín Núñez 2013), donde los hablantes quieren eximirse de cualquier responsabilidad ante lo expuesto en sus discursos y quitan relevancia a lo planteado con anterioridad gracias a estos marcadores.

Centrándonos específicamente en el grupo de marcadores con usos recapitulativos que aquí nos interesan, con la finalidad de presentar esta función pragmática hallamos las unidades que se anotan en el cuadro 2, con la frecuencia absoluta y porcentaje de frecuencia que en él se detallan.

\begin{tabular}{|l|l|l|}
\hline Marcador & Frecuencia absoluta & Porcentaje de frecuencia \\
\hline En fin & 126 & 39,5 \\
\hline O sea & 67 & 21,00 \\
\hline Total & 41 & 12,85 \\
\hline Bueno & 23 & 7,21 \\
\hline Vamos & 19 & 5,97 \\
\hline La verdad & 12 & 3,76 \\
\hline Al fin y al cabo & 9 & 2,82 \\
\hline En general & 8 & 2,51 \\
\hline Nada & 4 & 1,25 \\
\hline Al final & 3 & 0,94 \\
\hline En definitiva & 3 & 0,94 \\
\hline Vaya & 2 & 0,63 \\
\hline Después de todo & 1 & 0,31 \\
\hline Por lo general & 1 & 0,31 \\
\hline TOTAL & $\mathbf{3 1 9}$ & $\mathbf{1 0 0}$ \\
\hline
\end{tabular}

Cuadro 2. Frecuencias absolutas y porcentajes de uso de los reformuladores recapitulativos en el español de Granada

Observamos que es en fin el marcador con mayor frecuencia de uso, alcanzando cotas ligeramente por encima del resto, con lo cual podemos afirmar que los datos de Granada confirman que es el reformulador recapitulativo más característico del discurso oral. Lo siguen las formas o sea, total, bueno y vamos, como las de mayor significación. Podemos confirmar, por tanto, que con esta función se emplean asiduamente formas que las clasificaciones tradicionales, como la de Martín Zorraquino y Portolés Lázaro (1999), no contemplan en su inventario, normalmente formas comunes y frecuentes en el discurso oral a las que el hablante recurre en contextos diferentes según lo que quiera expresar.

A continuación, presentamos un ejemplo de cada uno de los marcadores arriba expuestos para que el lector pueda tener constancia de la función recapitulativa en su entorno y compruebe a través de las muestras que cada uno de ellos podría llegar a ser intercambiable con el otro, tal como predice la prueba de la conmutación antes mencionada. 


\section{En fin}

(1) $\mathrm{I}^{4}$ : [...] las demás ciudades también// he estado en Galicia// he estado// en Barcelona// en Gerona/ en fin/ he estado// en muchas capitales/ pero/// como ésa mm// ésa me/ me me me// me encantó/// es la que más/ me ha gustado (GRAN-H32-032)

\section{O sea}

(2) I: Pues el primero en la Carrera del Darro// pero/ no tengo recuerdos/ porque con tres meses/ nada// después en el barrio de Los Pajaritos / en la calle Tórtola/// que ahí estuvimos pues/ hasta que yo tenía once años/ ¿no fue? sí hasta el ochenta y tres/// y luego en Pintor Zuloaga// en la zona de Alhamar/// hasta que me casé// hasta hoy// desde los once/ hasta los treinta y dos ¿no? o treinta y uno ¿cuántos tenía yo cuando me casé? treinta y uno (risas) es que ni me acuerdo treinta y uno fue// sí sí sí// y ya está/ o sea yo he vivido// en tres sitios diferentes que yo recuerde solamente dos// porque en la Carrera del Darro yo era muy chica// y/// eso (GRAN-M12-022)

\section{Total}

(3) I: [...] digo "mira// en vez de darme tanto/ me cortáis/ por aquí o por allí/ en vez de dos/ es el cuatro por ciento/ el tres por ciento/ lo que sea/ y buscamos uno/ que me ayude/ y así ya es otra cosa"// yo voy haciendo las notas/ yo voy haciendo los// lo que haya que hacer/ pero luego el reparto de...// la lía de paquetes y el reparto/ que eran...// (simultáneo: E = claro) "... pues $/ / \mathrm{mira} /$ me parece bien"// total/ lo hicimos// y yo busqué/ un muchacho de/ de mi pueblo// y ahora cada vez que me ve/ se alegra mucho en fin// la vida// que vas rozando con personas que te lo agradecen// que a él le vino muy bien/ aquello// y que desde entonces pues/ está contento// [...] (GRAN-H32-033)

\section{Bueno}

(4) I: (tiempo: 30:20) a lo mejor cambiaría también/ pues claro// la pobreza norte sur/ la// las guerras// bueno que cambiaría todo el mundo/ imagino/ pero eso// eso// me parece que llevaría (GRAN-M22-029)

\section{Vamos}

(5) I: (tiempo: 20:49) nosotros/ somos lo que es mis padres y mis hermanos somos seis// luego por parte de mi madre son// con mis abuelos/// y mi madre y mis tíos// somos/ son cinco// por parte de mi padre también somos/// ahora quedamos/ uno dos tres/ cuatro/ cinco/// y bueno/ mi abuelo/ son nueve hermanos/// mi abuela son tres hermanos/ o sea que tenemos familia pf// un montón// y de primos/ primos segundos/ ¡vamos! que es una familia que para hacer un convite de una boda o algo/ (risas) es un desastre jvamos!// es... (GRANH11-038)

\section{La verdad}

(6) $\mathrm{I}$ : / entonces/ claro// (tiempo $=31: 59)$ antes de que le pase a mi hija algo/ pues que me pase a mí veinte mil veces/// pero/ es que eso/// te voy a decir que va respecto a cada

4 Abreviamos con la letra $i$ mayúscula el término informante, lo que denota que cada una de las palabras que aquí se exponen han sido recogidas de boca de los hablantes que conforman la muestra.

5 Seguimos el sistema de codificación propuesto por PreseEA y común a todos los equipos que lo forman. Así, para este ejemplo: GRAN (ciudad de Granada), H (Hombre), 3 (Generación 3.a), 2 (Instrucción 2, nivel medio) y 32 (informante n. ${ }^{\circ} 32$ ). 
persona/ a cada circunstancia/// no lo sé/// no lo sé// porque yo he visto gente/ de que/ nos hemos criado juntas/ chicas// y se han descarriado/ en ese hábito// (ruido = aclaración de voz) ¿qué le encuentran?// pues no lo sé// ¿por qué se meten?/// a lo mejor por problemas familiares/ a lo mejor por/ circunstancias// no lo sé/// no lo sé// la verdad que// más vale no saberlo (GRAN-M11-042)

\section{Al fin y al cabo}

(7) I: [...] claro siempre volvemos al final y que conste que me da igual/ no quiero meterme por si alguien se molesta conmigo ni con la Junta ni con vamos ni el PSOE ni el PP para mí son todos iguales son una pandilla de inútiles// porque al fin y al cabo lo lo único que hacen esta gente es carrera política// nadie se plantea entrar en política para estar cuatro años y luego/ [...] (GRAN-H23-09)

\section{En general}

(8) I: [...] el restaurante/ hay uno de confianza/ uno de total confianza porque los productos son de primera calidad/ y también me gusta ir de vez en cuando y en general/ bueno la cocina bien hecha $[\ldots]$ (GRAN-M33-017)

\section{Nada}

(9) I: Mi casa de Granada bueno es de mis padres/ es una casa que tiene tres pisos// $\mathrm{mm}$ en la planta baja donde está el salón mi cuarto y/ el patio y la cocina/ además un cuarto de baño/ la segunda planta tienen todos/ dormitorios/ tres/ y un cuarto de baño y arriba del to(do) está la terraza donde tenemos la lavadora/ tiene una una terraza muy grande/ con muchas macetas/ este verano $\mathrm{m}(\mathrm{e})$ ha toca(d)o a mí pintar la terraza que es la parte más grande de la casa/ y nada es una casa pues/ bastante grande. (GRAN-H13-02)

\section{Al final}

(10) I: y luego// cada día// se ha/ se ha/ $\mathrm{mm}$ ts fomentado más/ ese tipo de ocio/// (tiempo: 48:02) y ese tipo de ocio// si si cada día se fomenta más/ pues cada día lo controlamos más// al final// $\mathrm{mm}$ de no haber hecho bien el trabajo/ poco a poco// van a tener que tomar $\mathrm{mm}$...// decisiones radicales// que luego ni son socia-/ socialmente bien vistas ni nada porque ahora dirán// una solución/ podría ser// prohibir// absolutamente el consumo de alcohol// (GRAN-H22-027)

\section{En definitiva}

(11) I: Ts bueno/ yo pienso que están contentos/ ee el niño tuvo problemas al principio/ pienso por su carácter// y porque es un niño mu(palabra cortada)/ pienso yo que es muy inteligente// y es un niño al que le gustan cosas que a los niños normalmente no les gusta// en definitiva yo pienso que de cara a sus compañeros es un niño raro ¿eh?// que es un niño que le guste la astronomía y que le guste// (GRAN-H23-08)

\section{Vaya}

(12) I: Yo/ de esas cosas (fragmento ininteligible) (risas)// de mi cargo de mis/ cientos de cargos/ que estoy ya muy harto de cargos// la verdad/// que// no sé por qué/ me han caído tantas cosas.../ relacionadas siempre con cuestiones universitarias a las que yo no puedo decir// tan abiertamente que no// pero vaya yo creo que/ que ya es demasiado lo que tengo/ y creo que hay que empezar a/ soltar cosas// pero también la gente tiene que tirar para adelante y hacerse/ cargo de/ que hay 
que otras funciones que realizar no solamente/ investigar y dar clases// y estudiar/ hace falta también// estas labores que... tenemos que.../ repartirlas// vaya. (GRAN-H33-013)

\section{Después de todo}

(13) I: (...) y en el trayecto de la casa al instituto/ me quedé $\sin / /$ sin paraguas/// pues la verdad es que fue un año curioso/// pero/ lo pasé bien después de to(do)// porque estuve en un instituto donde la mayoría eran interinos era gente de paso// (GRAN-M23-010)

\section{Por lo general}

(14) I: bueno yo las arreglé como pude y la verdad es que pasé unas// un buen servicio militar/ independientemente de de lo que es la distancia ¿no? porque vine/ bastante poco// a la península/ o sea que yo vi a mis/ a mis hermanos crecer ¿no? cuando venía yo veía qu(e) habían// qu(e) habían creci(d)o// por lo general bien (GRAN-H23-09)

\subsection{Variación sociopragmática}

Como anunciábamos en el apartado dedicado a la metodología, es de esperar que, en hablantes con características y realidades tan diversas, el uso de unos marcadores concretos y no otros pueda verse alterado por circunstancias personales o por la duración e intensidad de cada conversación. Esto es apreciable en la figura número 2 que representa la dispersión en los hablantes. Podemos observar cómo se produce un aumento notable del uso de los reformuladores recapitulativos en el grupo de hablantes que va del número 6 al 12, correspondientes a la segunda generación del nivel alto, y al grupo que se sitúa entre los hablantes número 30 y 36, tercera generación del nivel de instrucción medio.

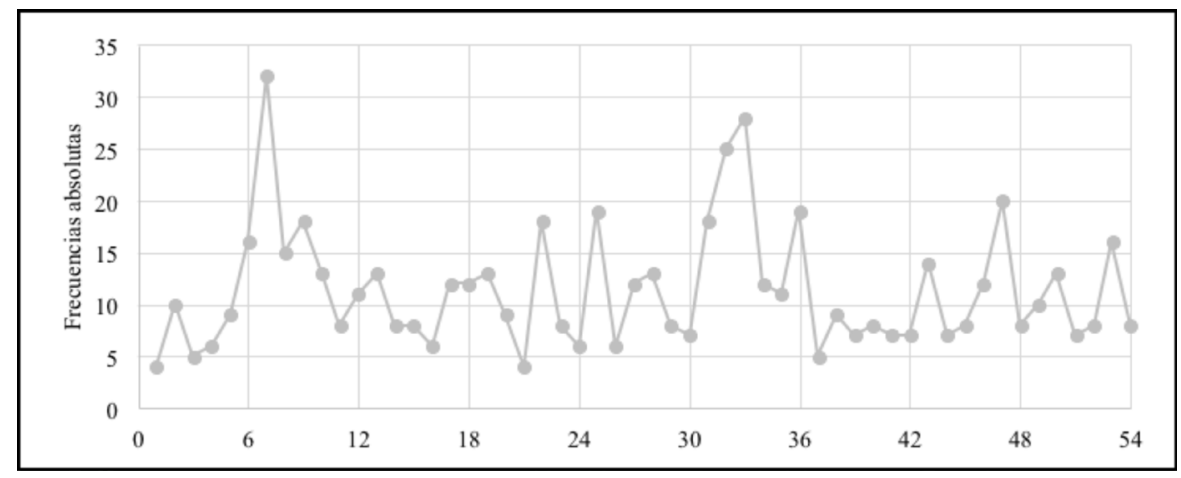

Fig. 2. Distribución del uso de reformuladores de recapitulación en el conjunto de hablantes de la muestra

Para evitar distorsiones en el análisis sociolingüístico, ponderamos los datos según el número absoluto de marcadores emitidos por cada interviniente y según la duración total de cada entrevista. Los datos resultantes sobre el uso de este grupo de marcadores discursivos con relación a las variables sociales seleccionadas son los que se detallan a continuación. 
En la figura número 3, podemos ver el uso de los recapitulativos según el sexo de los hablantes que los emplean y determinar que son los hombres los que mayor uso hacen de este tipo de partículas. Sin embargo, al analizar estadísticamente los datos obtenemos que $\mathrm{Chi}^{2}$ es 2,278 y p valor, 0,137 , con lo cual no podemos garantizar una relación de dependencia entre el uso de estas unidades y la variable social analizada.

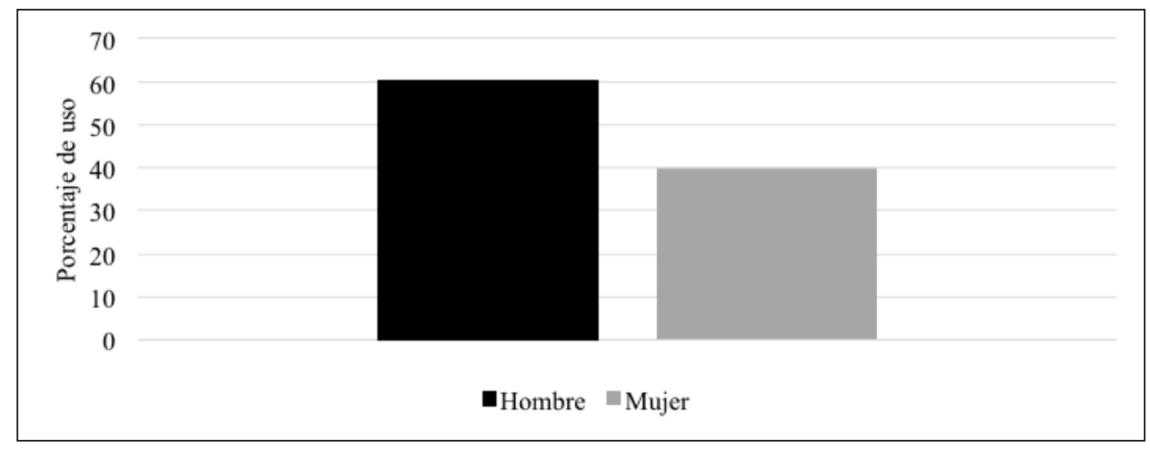

Fig. 3. Empleo de los recapitulativos en la ciudad de Granada según el sexo de los hablantes

En cuanto a la edad, la representación gráfica número 4, que se presenta seguidamente, denota el uso reducido de este tipo de unidades por parte de los hablantes de primera generación. Son, por tanto, los granadinos de segundo y tercer grupo etario quienes más emplean los recapitulativos. Sin embargo, al analizar los datos descubrimos un $\mathrm{Chi}^{2}$ de 1,696 y un $\mathrm{p}$ valor de 0,194 , que demuestran que no hay una relación directa entre la edad de los informantes y la frecuencia de uso de estas partículas.

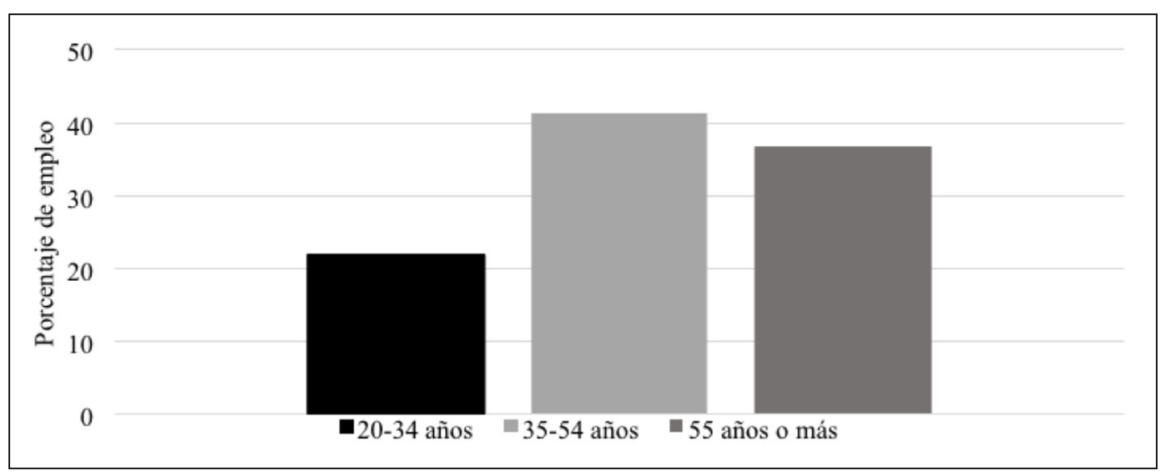

Fig. 4. Uso de los reformuladores de recapitulación según la edad de los granadinos

Por último, en cuanto al nivel de instrucción se refiere, notamos que también son, con diferencia, los hablantes de nivel medio y alto quienes más utilizan estos reformuladores, mientras los de nivel bajo apenas recurren a ellos. Así puede observarse en la figura 5. 


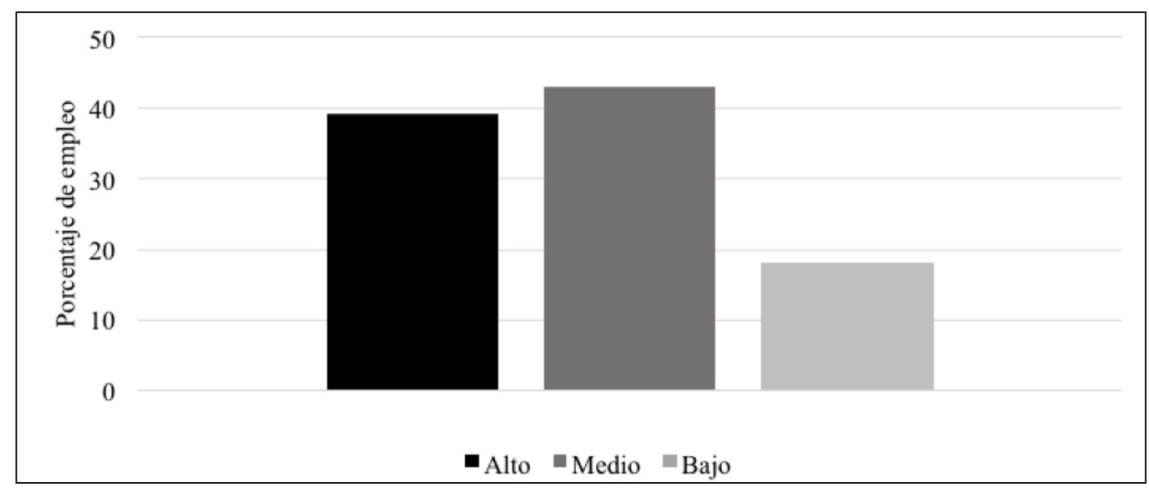

Fig.5. Uso de los recapitulativos en la ciudad de Granada según el nivel de instrucción

$\mathrm{Al}$ estudiar estadísticamente los valores, descubrimos un $\mathrm{Chi}^{2}$ de 3,181 y un p valor de 0,050, con lo cual esta relación se encuentra al límite de lo que hemos considerado significativo. Para confirmar la posible dependencia entre los niveles educativos y el empleo de los marcadores de recapitulación, llevamos a cabo la ANOVA de Kruskal Wallis, que arroja un $\mathrm{Chi}^{2}$ de 7,109 y un p valor de 0,029 , con lo cual sí que parece existir una correlación entre ambas variables.

Con lo que hemos visto, salvo en el caso del nivel educativo, no podemos determinar que exista relación expresa entre las variables sociales analizadas y el empleo de los reformuladores de recapitulación en el español de Granada. Por eso hemos procedido a cruzar las variables diastráticas entre sí mediante la construcción de tablas de contingencia para comprobar qué sucedía y los resultados hallados se muestran en las líneas siguientes.

En primer lugar, observamos en la figura número 6 la relación entre la edad y el sexo y la frecuencia de uso de estos marcadores. Vemos que la tendencia cambia claramente en el grupo de mujeres de primera generación, que hace un empleo mayor de los recapitulativos que los hombres. Seguramente, esto viene motivado por los cambios sociales a los que este grupo de féminas se han visto expuestas frente a sus progenitoras y también a su deseo de constituir y formar parte de una sociedad que las escucha y atiende lo que tienen que decir. El análisis de Chi cuadrado de Pearson y p valor demuestra que hay significación con unos resultados de 13,156 y 0,001 , respectivamente. 


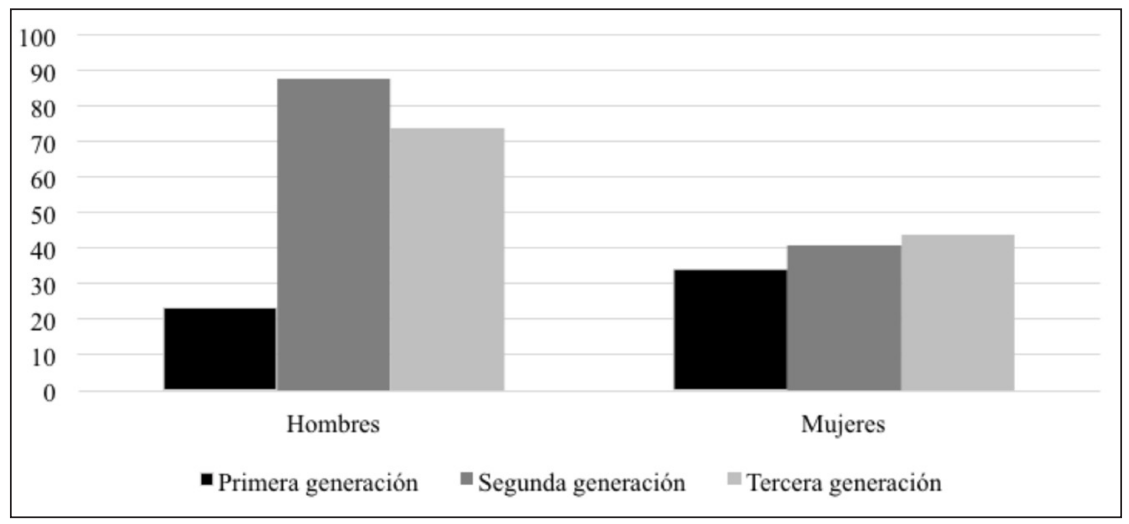

Fig.6. Empleo de los reformuladores recapitulativos según el sexo y la edad

En segundo lugar, encontramos la información para la relación entre el sexo y el nivel educacional de los hablantes. Vemos que en los hombres de nivel medio y alto el empleo de estos marcadores aumenta frente a las mujeres, mientras en el nivel bajo ambos sexos hacen un uso limitado de la recapitulación, posiblemente porque este grupo social tenga que enfrentar otras vicisitudes que lo haga no prestar tanta atención a la conclusión de su discurso. El análisis estadístico de Pearson revela un $\mathrm{Chi}^{2}$ de 6,0338 y un p valor de 0,048 , lo que demuestra que hay relación entre las variables y la frecuencia de uso. Así se aprecia en la representación 7.

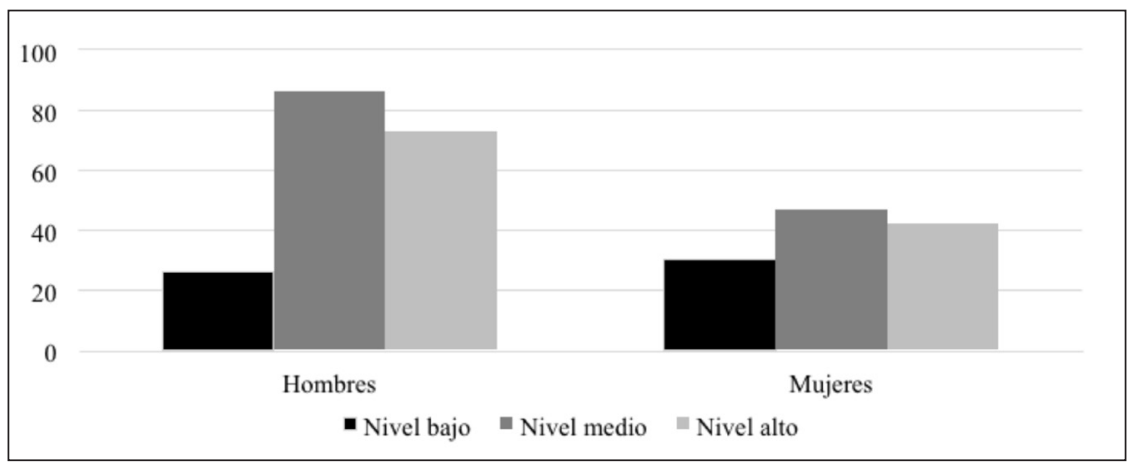

Fig. 7. Uso de los recapitulativos según el sexo y el nivel de instrucción

Por último, con relación a la edad y el nivel de estudios de los hablantes granadinos hemos encontrado unos resultados de $\mathrm{Chi}^{2}$ de 38,226 y p valor $<0,001$, que revelan la relación de dependencia entre el empleo de estas unidades y las variables sociales estudiadas. Si atendemos a la figura 8, podremos observar cómo lo común es que el pico de uso se produzca en la segunda generación, menos en el nivel educativo medio, donde son los hablantes longevos quienes emplean con mayor asiduidad este tipo de partículas, muy por 
encima del resto. Esto puede deberse a que es este grupo etario el que más conciencia tiene de la importancia de estructurar y concluir correctamente los discursos con vistas al oyente y porque el nivel medio busca constantemente reforzar su posición y asemejarse a los hablantes de nivel alto para sentirse más integrados.

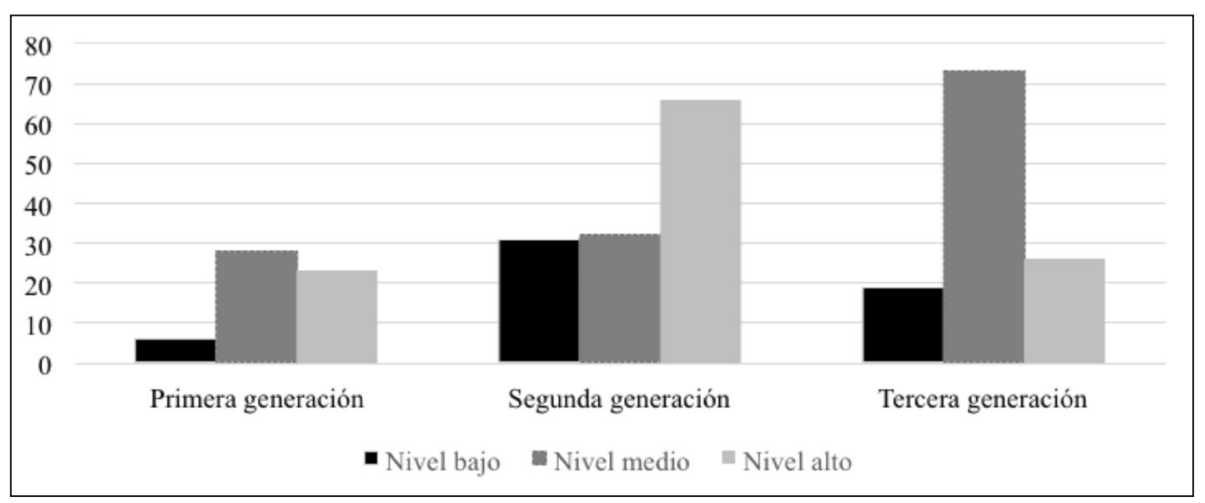

Fig. 8. Empleo de los recapitulativos según la edad y el nivel educativo

\subsection{Reformuladores recapitulativos más empleados}

Una vez presentados los hallazgos sobre los reformuladores de recapitulación en general, nos centraremos en este apartado en los datos relativos a los reformuladores más utilizados, esto es, en fin, o sea y total ${ }^{6}$.

En cuanto a en fin, su distribución se reparte tal como observamos en el cuadro número 4. En él se puede apreciar que el primer grupo etario prácticamente no lo utiliza en ninguno de los contextos de nuestro corpus, mientras las otras dos generaciones lo emplean con frecuencia. Algo similar ocurre con el nivel bajo, que hace una utilización reducida de esta partícula.

\begin{tabular}{|l|c|c|c|c|c|c|c|}
\cline { 2 - 7 } \multicolumn{1}{c|}{} & \multicolumn{2}{c|}{$\begin{array}{c}\text { Generación 1 } \\
\text { (20-34 años) }\end{array}$} & \multicolumn{2}{c|}{$\begin{array}{c}\text { Generación 2 } \\
\text { (35-54 años) }\end{array}$} & \multicolumn{2}{c|}{$\begin{array}{c}\text { Generación 3 } \\
\text { (más de 55 años) }\end{array}$} & \multirow{2}{*}{ Total } \\
\cline { 2 - 7 } \multicolumn{1}{c|}{} & Hombres & Mujeres & Hombres & Mujeres & Hombres & Mujeres & \\
\hline Nivel alto & 0 & 0 & 32 & 6 & 7 & 6 & 51 \\
\hline Nivel medio & 0 & 1 & 7 & 5 & 28 & 13 & 54 \\
\hline Nivel bajo & 0 & 0 & 0 & 10 & 3 & 8 & 21 \\
\hline Total & 0 & 1 & 39 & 21 & 38 & 27 & 126 \\
\hline
\end{tabular}

Cuadro 4. Distribución de en fin como reformulador recapitulativo en el PreseEA-Granada Al efectuar el análisis de la varianza (ANOVA), comprobamos que la edad presenta

6 Seguimos a San Martín Núñez (2014) y tomaremos como análisis aquellos usos en los que haya, al menos, 25 ocurrencias, para que la estadística sea lo más fiable posible. 
significación en el uso de este marcador, con unos valores de $\mathrm{Chi}^{2}$ de 4,950 y un p valor de 0,010. Para contrastar este resultado, realizamos la prueba no paramétrica o ANOVA de Kruskal Wallis, que arroja también significación, con un $\mathrm{Chi}^{2}$ de 17,075 y un p valor de 0,000. En la figura número 9 observamos cómo se reparten las medias estimadas de empleo de en fin, que se distribuyen en: primera generación $(0,06)$, segunda $(3,33)$ y tercera $(3,61)$, con lo cual su uso se ciñe principalmente a los hablantes mayores de la muestra.

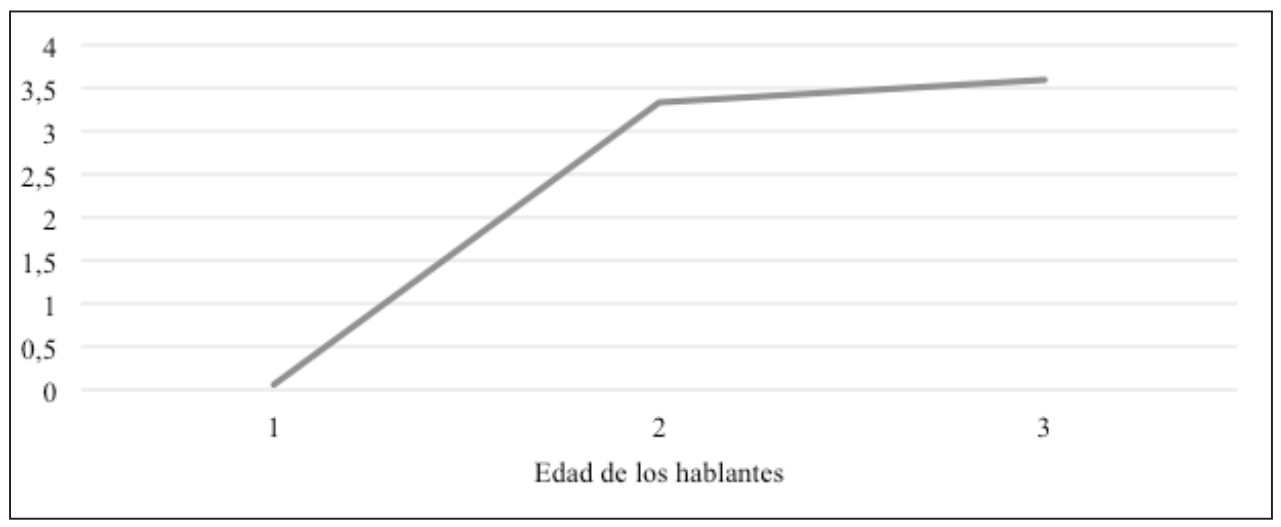

Fig. 9. Medias marginales estimadas de en fin

En cuanto a $o$ sea recapitulativo, los datos que hemos recopilado en el corpus PresEeA de Granada pueden leerse en el cuadro 5, que se expone a continuación.

Como podemos ver, su empleo es bastante homogéneo entre los diferentes grupos etarios y sexos, aunque el nivel de instrucción parece un punto de inflexión al mostrar datos muy bajos para los hablantes con estudios primarios o sin estudios.

\begin{tabular}{|l|c|c|c|c|c|c|c|}
\cline { 2 - 7 } \multicolumn{1}{c|}{} & \multicolumn{2}{c|}{$\begin{array}{c}\text { Generación 1 } \\
\text { (20-34 años) }\end{array}$} & \multicolumn{2}{c|}{$\begin{array}{c}\text { Generación 2 } \\
\mathbf{( 3 5 - 5 4} \text { años) }\end{array}$} & \multicolumn{2}{c|}{$\begin{array}{c}\text { Generación 3 } \\
\text { (más de 55 años) }\end{array}$} & \multirow{2}{*}{ Total } \\
\cline { 2 - 7 } \multicolumn{1}{c|}{} & Hombres & Mujeres & Hombres & Mujeres & Hombres & Mujeres & \\
\hline Nivel alto & 1 & 3 & 6 & 5 & 5 & 3 & 23 \\
\hline Nivel medio & 6 & 8 & 2 & 2 & 9 & 6 & 33 \\
\hline Nivel bajo & 3 & 1 & 4 & 2 & 1 & 0 & 11 \\
\hline Total & 10 & 12 & 12 & 9 & 15 & 9 & 67 \\
\hline
\end{tabular}

Cuadro 5. Resultados del uso de o sea como recapitulativo en Granada

La relación entre las variables sociales que estudiamos y la frecuencia de uso de o sea recapitulativo ha resultado no mostrar significatividad, tanto en la prueba paramétrica como en la no paramétrica. 
Con relación a total, el análisis arrojó los datos que se presentan en el cuadro 6. Comprobamos que los hablantes hombres de segunda y tercera generación son quienes mayor uso hacen de esta forma, mientras que en las mujeres resulta ser un marcador de escasa rentabilidad.

\begin{tabular}{|l|c|c|c|c|c|c|c|}
\cline { 2 - 7 } \multicolumn{1}{c|}{} & \multicolumn{2}{c|}{$\begin{array}{c}\text { Generación 1 } \\
\mathbf{( 2 0 - 3 4} \text { años) }\end{array}$} & \multicolumn{2}{c|}{$\begin{array}{c}\text { Generación 2 } \\
\mathbf{( 3 5 - 5 4} \text { años) }\end{array}$} & \multicolumn{2}{c|}{$\begin{array}{c}\text { Generación 3 } \\
\text { (más de 55 años) }\end{array}$} & \multirow{2}{*}{ Total } \\
\cline { 2 - 7 } & Hombres & Mujeres & Hombres & Mujeres & Hombres & Mujeres & \\
\hline Nivel alto & 1 & 1 & 0 & 1 & 0 & 0 & 3 \\
\hline Nivel medio & 2 & 3 & 7 & 0 & 15 & 1 & 28 \\
\hline Nivel bajo & 0 & 0 & 2 & 5 & 3 & 0 & 10 \\
\hline Total & 3 & 4 & 9 & 6 & 18 & 1 & 41 \\
\hline
\end{tabular}

Cuadro 6. Aparición del marcador total en el corpus granadino

Tras llevar a cabo la prueba de la varianza (ANOVA), hallamos unos resultados de Chi cuadrado de 3,587 y un p valor de 0,035 , ratificando que su uso está directamente relacionado con el nivel de instrucción de los hablantes, siendo mayor en los de nivel medio y después en los de nivel bajo. Apenas se encuentra, por tanto, en el nivel educativo superior porque este grupo no se identifica con él y lo percibe como una fórmula excesivamente coloquial e informal. Puede seguirse lo que hemos apuntado respecto a total en la representación gráfica número 10 , donde se presentan las medias marginales del marcador que se reparten en: nivel alto $(0,17)$, nivel medio $(1,56)$ y nivel bajo $(0,56)$.

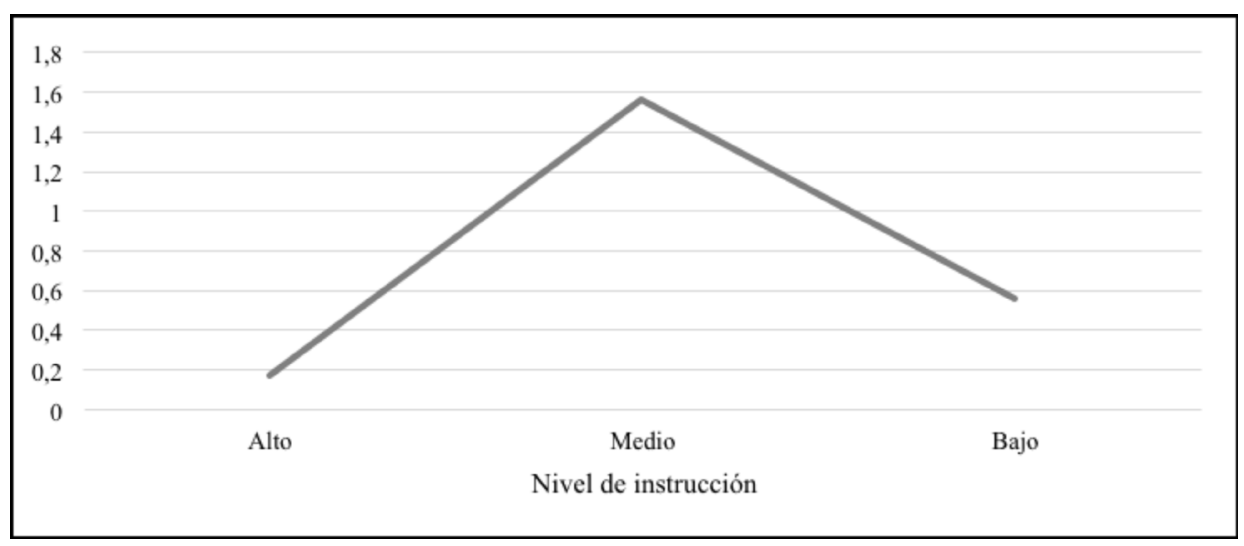

Fig. 10. Medias marginales estimadas de total

Sin embargo, con los datos que revela la prueba no paramétrica o ANOVA de Kruskal Wallis, que determina un $\mathrm{Chi}^{2}$ de 3,497 y un $\mathrm{p}$ valor de 0,174 , no podemos afirmar que exista correlación entre su frecuencia de uso y la variable nivel de instrucción. 


\section{CONCLUSIONES}

Con relación al análisis expuesto del funcionamiento pragmático-discursivo de los reformuladores con función recapitulativa en el español de Granada y su distribución diastrática, presentamos las conclusiones siguientes.

En primer lugar, encontramos que el porcentaje de uso de los reformuladores con este valor, dentro del conjunto total de marcadores que se dedican a la reformulación discursiva, es relativamente alto $(26,90 \%)$. Esta labor es efectuada por distintas unidades, más o menos gramaticalizadas, algunas de las cuales no forman parte de las clasificaciones tradicionales de este tipo de marcadores, como o sea, bueno, la verdad o nada, pero que, por su alta frecuencia de uso en el discurso oral, el hablante emplea en determinadas situaciones para presentar una síntesis de lo que ha expuesto, o numerado previamente, cuando se trata de varios elementos.

En segundo lugar, confirmamos que en fin es el reformulador recapitulativo por excelencia en la oralidad de los hablantes granadinos, cuyo uso, además, viene marcado por la edad de los informantes, pues cuanto mayores son, más utilizan esta partícula. Después, en orden de importancia, se presentan o sea y total. El primero no presenta variación en función de las variables sociales que consideramos, más bien se reparte de forma equilibrada entre las celdas del corpus. El segundo, sin embargo, manifiesta, al menos en la prueba paramétrica, una relación de dependencia entre el nivel educativo del hablante y su frecuencia de empleo, siendo este marcador apenas utilizado por parte de los hablantes con estudios superiores.

Sería conveniente prestar atención a lo que ocurre en otras comunidades de habla hispana que han estudiado este tipo de marcadores desde una perspectiva sociolingüística, tales como Sevilla o Santiago de Chile, lo cual, además, nos serviría para cumplir con una de las máximas del proyecto Preseea, que es el intercambio científico y la comparabilidad de resultados entre los equipos de investigación que lo integran.

Otra línea de investigación que plantemos es conocer el entorno sintáctico que rodea a cada uno de estos elementos para comprobar qué influencia ejercen otros marcadores, las conjunciones o las pausas en la función pragmática de la unidad. Además, nos interesa estudiar en qué tipo de actos discursivos es más frecuente el empleo de los reformuladores recapitulativos, aunque, de partida, intuimos que será mayor en aquellas secuencias en las que el hablante enumera una serie de hechos o acciones, como en el acto narrativo, o de adjetivos calificativos, como ocurre en las descripciones de personas, paisajes u objetos.

\section{REFERENCIAS BIBLIOGRÁFICAS}

Adam, J. M. y F. Revaz (1989). "Aspects de la structuration du texte descriptif: les marqueurs d'énumération et de reformulation", $L a F, 81$, pp. 59-98: https://www.persee.fr/doc/lfr_00238368_1989_num_81_1_4769 (25-04-2019).

Briz Gómez, A. (2014 [2001]). El español coloquial en la conversación: esbozo de pragmagramática. Barcelona: Ariel.

Briz Gómez, A., Pons Bordería, S. y J. Portolés Lázaro (coords.). 2008. Diccionario de partículas discursivas del español: www.dpde.es (19-04-2019).

Carbonero Cano, P. y J. Santana Marrero (2010). "Marcadores del discurso, variación dialectal y variación social”. En Loureda, Ó. y E. Acín (coords.). Marcadores del discurso, hoy. Madrid: Arco Libros, pp. 497-522. 
Cortés Rodríguez, L. (2008 [1998]). "Marcadores del dscurso y análisis cuantitativo". En Martín Zorraquino, M. A. y E. Montolío Durán (eds.). Los marcadores del discurso. Teoría y análisis. Madrid: Arco Libros, pp. 143-160.

Cuartas López, L. C. (2011). Marcadores discursivos en el habla de la ciudad de Cartagena de Indias. Tesis doctoral, Universidad Nacional de Colombia: http://www.bdigital.unal.edu. co/4263/1/448179.2011.pdf (23-05-2019).

Domínguez García, N. (2014). "Marcadores de cierre con la raíz fin-: criterios para un uso adecuado", RedELE: Revista Electrónica de Didáctica ELE, 26: https://sede.educacion.gob.es/publiventa/ detalle.action?cod=20207 (24-05-2019).

Domínguez García, N. (2016). Organizadores del discurso. Madrid: Arco Libros.

Domínguez, C. L. y A. Álvarez (2005). "Marcadores en interacción: un estudio de marcadores en el español hablado en Mérida (Venezuela)", Revista Virtual de Estudos da Linguagem - ReVEL, 3 (4): www.revel.inf.br (12-04-2019).

Figueras Solanilla, C. (2000). "Reflexiones en torno a las estrategias de reformulación parafrástica en la oralidad y en la escritura": https://cvc.cervantes.es/ensenanza/biblioteca_ele/asele/pdf/11/11_0297. pdf (22-05-2019).

Fuentes Rodríguez, C. (1990). "Procedimientos intradiscursivos: decir y los explicativos". En Carbonero Cano, P. y M. T. Palet Plaja (eds.). Sociolingüística andaluza 5. Habla de Sevilla y hablas americanas. Sevilla: Publicaciones de la Universidad, pp. 103-123.

Fuentes Rodríguez, C. (2009). Diccionario de conectores y operadores del español. Madrid: Arco Libros.

Fuentes Rodríguez, C. (2015). La sintaxis de los relacionantes supraoracionales. Madrid: Arco Libros.

Garcés Gómez, M. P. (2003). "Los marcadores de recapitulación y de reconsideración en el discurso", Revista de Investigación Lingüistica, 1(6), pp. 111-141: https://revistas.um.es/ril/article/ view/5531/5391 (27-05-2019).

Garcés Gómez, M. P. (2006a). "Marcadores del discurso y actos de habla". En Casado Velarde, M., González Ruiz, R. y M. V. Romero Gualda (coords.). Análisis del discurso: lengua, cultura, valores: Actas del I Congreso Internacional. Madrid: Arco Libros, vol. 2, pp. 1311-1324.

Garcés Gómez, M. P. (2006b). "La evolución de los marcadores de ordenación discursiva en español", Romanistiches Jahrbuch, 57, pp. 327-351. DOI: 10.1515/9783110192889.2.327.

Garcés Gómez, M. P. (2008). La organización del discurso: marcadores de ordenación y de reformulación, Madrid/Frankfurt: Iberoamericana/Vervuert: http://ebookcentral.proquest.com/lib/ ugr/reader.action?docID=3215272\&query $=(15-06-2019)$.

Garcés Gómez, M. P. (2014). Diacronía de los marcadores discursivos y representación en un discurso histórico. A Coruña: Universidad de A Coruña.

Garcés Gómez, M. P. (2017). "La reformulación discursiva y los procesos de recapitulación y conclusión: a propósito de los marcadores en fin y total", Romanische Forschungen, 129 (3), pp. 295-316. DOI: 10.3196/003581217821694319

González Mafud, A. y M. Perdomo Carmona (2018). “Aproximación al estudio de los marcadores discursivos en muestras de habla culta de La Habana", Universidad de La Habana, 285, pp. 25-49.

Guirado Zapata, K. (2019). "Reformulación locutiva/ilocutiva: o sea y es decir en una microdiacronía de la oralidad caraqueña”. Ponencia presentada en el Discourse Markers in Romance Language 6, Bérgamo, Italia.

Herrera Soler, H., Martínez Arias, M. R. y M. Amengual Pizarro (2011). Estadística aplicada a la investigación lingüistica. Madrid: EOS.

Jorgensen, A. M. y J. A. Martínez López. (2007). "Los marcadores del discurso del lenguaje juvenil de Madrid”, Revista Virtual de Estudos Da Linguagem, 5(9): www.revel.inf.br (28-03-2019).

Labov, W. (1972). "Some principles of Linguistic Methodology", Language in Society, 1, pp. 97-120.

Labov, W. (1983). Modelos sociolingüísticos. Madrid: Cátedra. 
Lavandera, B. (1978). "Where does the sociolinguistic variable stop?", Language in Society, 7, pp. 171-182.

Martí Sánchez, M. (2013). Los marcadores discursivos. Para estudiantes y profesores de español como lengua extranjera. Madrid: Edinumen.

Martín Butragueño, P. (2003). "Hacia una descripción prosódica de los marcadores discursivos. Datos del español de México". En Herrera Zendejas, E. y P. Martín Butragueño (eds.). La tonía. Dimensiones fonéticas y fonológicas. México: Colegio de México, pp. 375-402.

Martín Zorraquino, M. A. (1991). "Elementos de cohesión en el habla de Zaragoza". En Enguita Utrilla, J. M. (ed.). I Curso de Geografía Lingüistica de Aragón. Zaragoza: Institución Fernando el Católico, pp. 253-286.

Martín Zorraquino, M. A. y J. Portolés Lázaro (1999). "Los marcadores del discurso". En: Ignacio Bosque y Violeta Demonte (coords.). Gramática descriptiva de la lengua española. Madrid: Espasa Calpe, vol. 3, pp. 4051-4214.

Martínez Matos, H. y C. L. Domínguez (2006). “Análisis prosódico de algunos marcadores discursivos en el habla de Mérida, Venezuela", Lingüística de Español Actual, 28(2), pp. 247-264.

Moreno Fernández, F. (1996). "Metodología del «Proyecto para el Estudio Sociolingüística del Español de España y de América» (Preseea)”, Lingüística, 8, pp. 257-287.

Moreno Fernández, F. (2015 [1998]). Principios de sociolingüistica y sociología del lenguaje. Barcelona: Ariel.

Moya Corral, J. A. (coord.). (2007). El español hablado en Granada. Corpus oral para su estudio sociolingüístico (Nivel de estudios alto). Granada: Editorial Universidad de Granada.

Murillo Ornat, S. (2016). "Sobre la reformulación y sus marcadores", Cuadernos AISPI, 8, pp. 237258: https://dialnet.unirioja.es/servlet/articulo?codigo=6249639 (10-09-2019).

Poblete Bennett, María Teresa y Gladys Cepeda (1997). "Los marcadores discursivo-conversacionales en el habla femenina de Valdivia", Boletín de Filología (Universidad de Chile), XXXVI, pp. 2535: https://boletinfilologia.uchile.cl/index.php/BDF/article/view/21480/22778 (15-06-2019).

Poblete Bennett, M. T. y G. Cepeda (2006). "Cortesía verbal, modo y modalidad. Los marcadores discursivos”, Revista Signos, 39(62), pp. 357-377. DOI: 10.4067/S0718-09342006000300002

Portolés Lázaro, J. (2004). Pragmática para hispanistas. Barcelona: Síntesis.

Portolés Lázaro, J. (2014 [1998]). Marcadores del discurso. Barcelona: Ariel.

Rincón, L. M. (2013). "Variación en los marcadores del discurso en el habla de Bucaramanga", Íkala. Revista de Lenguaje y Cultura, 18(2), pp. 17-35: http://www.scielo.org.co/pdf/ikala/v18n2/ v18n2a2.pdf (10-03-2019).

Rodríguez Cadena, Y. (1999). "Marcadores discursivos en el habla de Barranquilla", Litterae, 8, pp. 197-221.

Rojas Inostroza, C., Rubio Núñez, A., San Martín Núñez, A. y S. Guerrero González (2012). "Análisis pragmático y sociolingüístico de los marcadores discursivos de reformulación en el habla de Santiago de Chile". Lenguas Modernas, 40, pp. 103-124: https://revistas.uchile.cl/index. $\mathrm{php} / \mathrm{LM} /$ article/view/30767/32513 (20-06-2019).

Ruiz González, N. (2018). "La reformulación discursiva en español: el caso de o sea", Itinerarios: Revista de estudios lingüísticos, literarios, históricos y antropológicos, 28, pp. 177-199: http:// itinerarios.uw.edu.pl/itinerarios-no-28/ (22-04-2019). DOI: 10.23825/ITINERARIOS.28.2018.09

Said-Mohand, A. (2014). "Los marcadores del discurso en el español hablado en los Estados Unidos: Estado de la cuestión”, Tonos Digital: Revista Electrónica de Estudios Filológicos, 26: http:// www.um.es/tonosdigital (28-11-2017).

San Martín Núñez, A. (2013). "Los reformuladores de distanciamiento en el corpus PreseEA de Santiago de Chile", Boletín de filología: (Universidad de Chile), 48 (1), pp. 171-199: https:// boletinfilologia.uchile.cl/index.php/BDF/article/view/27141 (25-03-2019). DOI: 10.4067/S071893032013000100008 
San Martín Núñez, A. (2014). Variación sintáctica y discursiva en el español hablado en Santiago de Chile: análisis sociolingüistico del queísmo, el dequeísmo, el discurso referido y los marcadores de reformulación. Tesis doctoral, Universidad de Valladolid: http://uvadoc.uva.es/bitstream/ handle/10324/15865/Tesis746-160210.pdf?sequence=1\&isAllowed=y (07-08-2019).

San Martín Núñez, A. (2015). "Variantes y equivalentes funcionales de al final: los reformuladores de recapitulación en el habla santiaguina", Revista de Lingüistica Teórica y Aplicada, 53(2), pp. 97-119: https://scielo.conicyt.cl/scielo.php?script=sci_arttext\&pid=S0718-48832015000200005 (11-06-2019). DOI: 10.4067/S0718-48832015000200005

San Martín Núñez, A. (2016a). "Análisis sociolingüístico de los reformuladores de rectificación en el habla santiaguina". Literatura y lingüística, 33, pp. 241-264. DOI: 10.4067/S071658112016000100012

San Martín Núñez, A. (2016b). "Los marcadores de reformulación en el español oral de Santiago de Chile: análisis discursivo y sociolingüístico", Oralia: Análisis del discurso oral, 19, pp. 281-322.

San Martín Núñez, A. (2017). "Análisis sociolingüístico de los reformuladores de explicación en el español hablado de Santiago de Chile", Revista Signos, 50 (93), pp. 124-147: https://scielo.conicyt. cl/scielo.php?script=sci_arttext\&pid=S0718-09342017000100006 (23-07-2019). DOI: 10.4067/ S0718-09342017000100006

San Martín Núñez, A. y S. Guerrero González (2016). "Los marcadores de reformulación en el corpus Preseea de Santiago de Chile", Forma y función, 29 (2), pp. 15-38: https://dialnet.unirioja.es/ servlet/articulo?codigo=6403631 (31-07-2019). DOI: 10.15446/fyf.v29n2.60187

Santana Marrero, J. (2017). "Marcadores del discurso y variación diastrática: análisis en el sociolecto bajo". En Marta León-Castro y Adamantia Zerva (coords.): Sociolingüística andaluza 17. La variación en el español actual. Estudios dedicados al profesor Pedro Carbonero. Sevilla: Publicaciones de la Universidad, pp. 289-313.

Santos Río, L. (2003). Diccionario de partículas. Salamanca: Luso-Española.

Serna Pinto, M. A. y N. Hernández Miranda (2016). Los marcadores discursivos en el habla de la ciudad de Pereira con base en el corpus del Proyecto para el Estudio Sociolingüístico del Español de España y América - Preseea: http://repositorio.utp.edu.co/dspace/bitstream/ handle/11059/6519/418S486.pdf?sequence=1\&isAllowed=y (08-07-2019). Trabajo final de grado, Universidad Tecnológica de Pereira.

Silva-Corvalán, C. y A. Enrique-Arias (2017[2001]). Sociolingüística y Pragmática del español. Washington D.C: Georgetown University Press.

Taibo Cao, L. (2016). Los marcadores del discurso en el habla culta de Montevideo: análisis del corpus Preseea. Trabajo final de máster, Universidad Nacional de Educación a Distancia: http://espacio.uned.es/fez/eserv/bibliuned:master-Filologia-AGyEE-Lmtaibo/Taibo_Cao_Luis_Marcelo_ TFM.pdf (12-09-2019).

Travis, C. (2005). Discourse markers in colombian Spanish: A study in polysemy. Berlin: Mouton de Gruyter.

Valencia Espinoza, A. (ed.). (2014). Marcadores discursivos en la norma culta hispánica (1964-2014). Cuadernos de la ALFAL, 5: https://mundoalfal.org/es/content/cuadernos-de-la-alfal-n\%C2\%BA5 (20-08-2019).

Valencia Espinoza, A. y A. Vigueras Ávila (eds.). (2015). Marcadores hispánicos. Usos de España y América en el corpus de estudio de la norma culta. México: UNAM.

Vásquez Cantillo, A. (2005). Los marcadores discursivos en la comunidad de habla de Barranquilla. Tesis de maestría, Instituto Caro y Cuervo.

Vásquez Cantillo, A. (2009). "Análisis sociolingüístico de los marcadores discursivos en la comunidad de habla barranquillera”, Cuadernos de Lingüística Hispánica, 13, pp. 43-66: https://dialnet. unirioja.es/servlet/articulo?codigo=3322139 (03-09-2019).

Vázquez Veiga, N. (2011-2012). "El tratamiento lexicográfico de los marcadores del discurso", Linred: Lingüistica en la Red, 9: https://ebuah.uah.es/dspace/handle/10017/24165 (06-04-2019). 